\title{
Classically Continuous Lattice Fluid Theory for Pure Polymeric Liquids
}

\author{
Hae Young JUNG \\ Department of Chemistry, DukSung Women's University, Ssang-Moon-Dong 419, \\ Do-Bong-Ku, Seoul 132-714, Korea
}

(Received April 11, 1996)

\begin{abstract}
The concept of a continuous lattice from the viewpoint of classical mechanics was introduced and a Lennard-Jones 6-12 potential was used to modify the lattice fluid theory of Sanchez and Lacombe. Thus, a new equation of state for pure polymeric liquids was obtained. With this equation, $P V T$ data, thermal expansivities and isothermal compressibilities of several polymeric liquids were calculated and compared with experimental values and with those of the lattice fluid theory and other theories. The new equation gave similar precision to Hole theory in the calculation of volume, thermal expansivity and isothermal compressibility and gave better agreement with the experimental data than the other theories used in the comparison.
\end{abstract}

KEY WORDS Equation of State / Lattice Theory /

Many theories have been suggested to describe the liquid state of polymers. One concept, widely used to describe the polymeric state, is the lattice. ${ }^{1}$ LF (lattice fluid) theory ${ }^{2,3}$ of Sanchez and Lacombe, which gains considerable success in describing polymeric liquids, is a good example. In the theory, sites on the lattice are discretely distributed and one monomeric segment occupies only one site on the lattice and interaction potential between segments is assumed to be constant and independent of distance. However, if we divide the lattice so finely that the distribution of sites on the lattice can be approximated to be classically continuous, it becomes possible to use the van der Waals and LennardJones 6-12 potential which are functions of distance or volume. Then, unlike the original lattice model, one monomer unit occupies many sites on the lattice as shown in Figure 1. In this work, dividing the lattice as above, we made continuous distribution of sites and used Lennard-Jones 6-12 potential to obtain the new equation of state for polymeric liquids.

\section{THEORETICAL}

Ways to Place $N_{1}$ r-mers on $N$ Sites of the Continuous Lattice

We can get the number of ways in the similar manner to Flory's approximation ${ }^{1}$ for polymer solution though one monomer unit occupies many sites and vacant sites exist, which will be seen in the following. Assume that one monomer unit, on the average, occupies $n$ sites as shown in Figure 1. Then.

$$
N=N_{0}+r N_{1} n
$$

where $N_{0}$ is the total number of vacant sites and,

$$
V=\left(N_{0}+r N_{1} n\right) v
$$

where $V$ is total volume and $v$ is volume occupied by one site on the lattice. In this work, it is assumed that $v$ is constant and so fine as to make the sites distributed continuously in terms of classical mechanics.

Taking into account that $r$ is negligible in comparison with $N / n$ and following Flory's approximation, $W$, the total number of ways to place all the $r$-mers on the lattice is,

$$
\begin{aligned}
\ln W= & N_{1} \ln \left[\frac{s\left(s-n_{\mathrm{s}}\right)^{r-2} n}{\sigma \mathrm{e}^{r-1}}\right]-\frac{N_{0}}{n} \ln \left(\frac{N_{0}}{N}\right) \\
& -N_{1} \ln \left(\frac{N_{1}}{N / n}\right)
\end{aligned}
$$

where $s$ is the maximum number of sites for the second monomer unit of a $r$-mer when the first monomer unit is placed, which is different from the number of nearest neighboring units of the first unit, and $n_{\mathrm{s}}$ is the number of sites already occupied by the first unit, among the $s$ sites for the third unit when the second unit is placed, and $\sigma$ is the symmetry number of the polymer. This situation is illustrated in Figure 1. The parameters, $s, n_{\mathrm{s}}$, and $n$, are introduced merely to make the sites classically continuous and it is not necessary to know the actual values of the parameters since this would have no effect

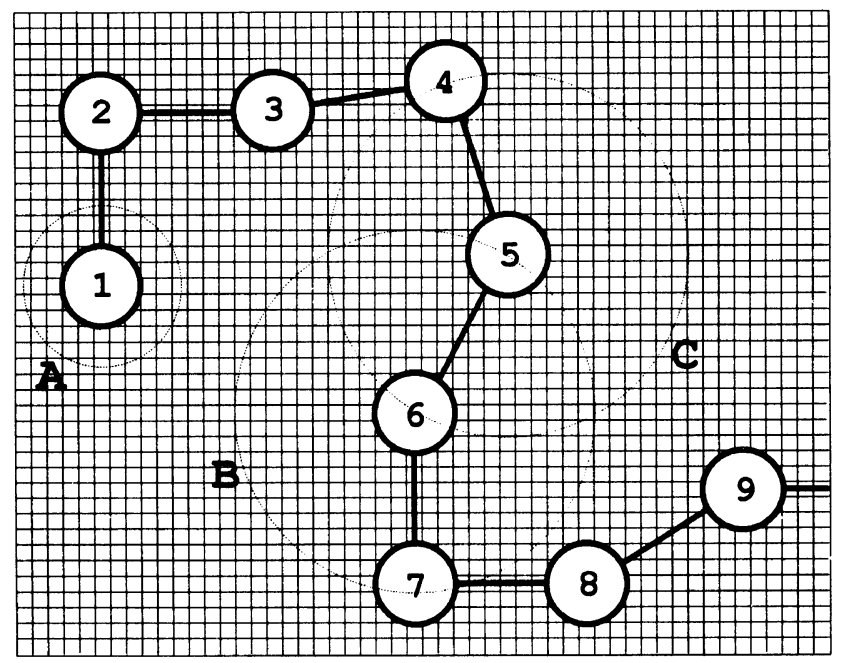

Figure 1. Schematic illustration of the distribution of one polymer and vacant sites on an approximately continuous lattice. Solid rings, numbered successively, indicate monomer units of the polymer and lattice points unoccupied by the rings are vacant sites. $n$ in eq 1 to 3 approximates the number of sites inside a dotted ring $\mathrm{A}$ around the first unit. $s$ in eq 3 approximates the number of sites on a dotted ring $\mathrm{B}$ around the sixth unit. $n_{\mathrm{s}}$ in eq 3 approximates half of the number of sites on the part of the ring $\mathrm{B}$ which is located inside a dotted ring $\mathrm{C}$ around the fifth unit. 
on the equation of state.

\section{New Equation of State from the Continuous Lattice Fluid} Theory

Since the sites on the lattice were made to be continuously distributed in terms of classical mechanics, we can use such volume-dependent potentials as the van der Waals or Lennard-Jones 6-12 potential. Combining eq 3 of the configurational entropy with the energy term of the van der Waals potential to make a canonical partition function and using the relationship of statistical mechanics between pressure and partition, we obtain the same equation of state as the original LF theory, ${ }^{2,3}$ as follows.

$$
\tilde{\rho}^{2}+\tilde{P}+\tilde{T} \tilde{\Gamma}[\ln (1-\tilde{\rho})+(1-1 / r) \tilde{\rho}]=0
$$

In eq $4, \tilde{T}=T / T^{*}$ and $T^{*}=\varepsilon^{*} / k$ and $\tilde{P}=P / P^{*}$ and $P^{*}=r N_{1} \varepsilon^{*} / V^{*}$ and $\tilde{\rho}=1 / \tilde{V}$, where $\varepsilon^{*}$ is the energy parameter in the van der Waals potential, and the other parameters have the same meaning as in the LF theory.

Equation 4 shows that the continuous LF theory with the van der Waals potential produces the same equation of state as that of the original LF theory.

According to Dee and Walsh, ${ }^{4,5}$ the cell theory using the potential of eq 5 , which assumes that all the cells of monomer units are distributed as in the face-centered cubic state, fits the $P V T$ data of a pure polymer and the partial miscibility of the polymer blend to experimental data better than Flory's equation of state theory using the van der Waals potential.

$$
\phi(\tilde{V})=\varepsilon^{*}\left(\frac{B}{\tilde{V}^{4}}-\frac{2 A}{\tilde{V}^{2}}\right)
$$

where $A=1.2045, B=1.011$, and $\varepsilon^{*}$ is the energy parameter of the Lennard-Jones 6-12 potential. Based on the work of Dee and Walsh, the author used the potential of eq 5 in this work. That is, the author assumes that all monomer units are on the average distributed as in the face-centered cubic state. But, since the cell theory is originally proposed to explain the liquid state rather than polymer in terms of molecular motion within cells, eq 5 is considered for a polymer as an adhoc potential rather than a potential of definite physical meaning.

With eq 5 , we obtain the following equation of state in the same manner as eq 4.

$$
4\left(A \tilde{\rho}^{3}-B \tilde{\rho}^{5}\right)+\tilde{P}+\tilde{T}[\ln (1-\tilde{\rho})+(1-1 / r) \tilde{\rho}]=0
$$

Since $r$ is very large for polymers, eq 6 becomes as follows.

$$
4\left(A \tilde{\rho}^{3}-B \tilde{\rho}^{5}\right)+\tilde{P}+\tilde{T}[\ln (1-\tilde{\rho})+\tilde{\rho}]=0
$$

From eq 7 , if we assume that $P^{*}, V^{*}$, and $T^{*}$ are constant, the thermal expansivity $\alpha$ and isothermal compressibility $\beta$ are obtained as,

$$
\begin{gathered}
T \alpha=\frac{4\left(A \tilde{\rho}-B \tilde{\rho}^{3}\right)+\tilde{P} / \tilde{\rho}}{4\left(-3 A \tilde{\rho}+5 B \tilde{\rho}^{3}\right)+\tilde{T} /(1-\tilde{\rho})} \\
P^{*} \beta=\frac{1}{\tilde{\rho}^{2}\left[4\left(-3 A \tilde{\rho}+5 B \tilde{\rho}^{3}\right)+\tilde{T} /(1-\tilde{\rho})\right]}
\end{gathered}
$$

\section{RESULTS AND DISCUSSION}

Using eq 7, PVT data of several polymeric liquids were calculated and compared with those of the LF theory, ${ }^{2,3}$ cell theory, ${ }^{4,5}$ Hole theory, ${ }^{6}$ and Flory's equation of state theory. ${ }^{7}$ In the calculations, it is assumed that parameters $P^{*}, V^{*}, T^{*}$ in each theory are constant. But they are not constant over a wide range of temperature and pressure. Here, as shown in Table I, the temperature range is taken to be less than $40^{\circ} \mathrm{C}$ and the pressure range is from 1 to $200 \mathrm{bar}$. The parameters were determined by minimizing the following root mean square of relative volume errors.

$$
R M S E=\sqrt{\frac{\sum_{i=1}^{N_{\text {data }}}\left(1-V_{\text {calc }} / V_{\text {expt1 }}\right)_{i}^{2}}{N_{\text {data }}}} \times 100 \%
$$

where $N_{\text {data }}$ is the number of experimental $P V T$ data. The results are listed in Tables I to $\mathrm{V}$, which show that this work gives better agreement with the experimental $P V T$ data than other theories except the Hole theory.

To calculate the experimental thermal expansivity $\alpha$, $P V T$ data were fitted to the following equation through linear regression of $\ln V$ with $t$.

$$
V(t)=V_{0} \exp (-\alpha t)
$$

where $t$ is Celsius temperature.

For calculation of the experimental isothermal compressibility, PVT data were fitted to the experimental

Table I. Results of $P V T$ calculations by eq $7^{\text {a }}$

\begin{tabular}{llllclll}
\hline Material & $T^{*} / \mathrm{K}$ & $P^{*} /$ bar & $V^{*} / \mathrm{cm}^{3} \mathrm{~g}^{-1}$ & $\begin{array}{c}\text { Temp } \\
\text { range } /{ }^{\circ} \mathrm{C}\end{array}$ & \multicolumn{2}{c}{$\begin{array}{c}\text { Pressure } \\
\text { range/bar } \% \text { error }\end{array}$} \\
\hline PMMA & 347.6 & 3585 & 0.7374 & $124.5-159.0$ & $1-200$ & 0.00604 \\
PcMA & 337.0 & 3260 & 0.7890 & $122.7-158.2$ & $1-200$ & 0.0109 \\
PnBMA & 299.7 & 3358 & 0.8241 & $33.9-73.5$ & $1-200$ & 0.0103 \\
BPE & 296.8 & 2649 & 1.023 & $135.1-160.5$ & $1-200$ & 0.00745 \\
LPE & 288.6 & 2839 & 1.008 & $152.9-180.9$ & $1-200$ & 0.0135 \\
PVA & 271.3 & 3695 & 0.7156 & $40.0-55.0$ & $1-200$ & 0.00575 \\
PS & 366.4 & 2765 & 0.8459 & $115.4-140.2$ & $1-200$ & 0.0107 \\
POMS & 373.2 & 2852 & 0.8614 & $149.4-179.2$ & $1-200$ & 0.0115
\end{tabular}

average $\%$ error $=0.00953$

${ }^{a}$ Ranges of temperature and pressure in other tables are the same as in Table I. PMMA, ${ }^{9}$ poly(methy! methacrylate); PcMA, ${ }^{9}$ poly(cyclohexyl methacrylate); PnBMA,${ }^{9}$ poly( $n$-butyl methacrylate); BPE, ${ }^{9}$ branched polyethylene; LPE, ${ }^{9}$ linear polyethylene; PVA, ${ }^{10}$ poly(vinyl acetate); $\mathrm{PS},{ }^{8}$ polystyrene (atactic); POMS,${ }^{8}$ poly( ortho-methyl styrene).

Table II. Results of PVT calculations by the lattice fluid theory ${ }^{\mathrm{a}}$

\begin{tabular}{lcccc}
\hline Material & $T^{*} / \mathrm{K}$ & $P^{*} /$ bar & $V^{*} / \mathrm{cm}^{3} \mathrm{~g}^{-1}$ & $\begin{array}{l}\text { Volume } \\
\% \text { error }\end{array}$ \\
\hline PMMA & 749.6 & 5000 & 0.8018 & 0.0121 \\
PcMA & 732.8 & 4588 & 0.8595 & 0.0139 \\
PnBMA & 624.3 & 4582 & 0.8895 & 0.0201 \\
BPE & 670.1 & 3865 & 1.125 & 0.0174 \\
LPE & 660.6 & 4245 & 1.113 & 0.0211 \\
PVA & 583.2 & 5139 & 0.7776 & 0.0116 \\
PS & 761.8 & 3745 & 0.9127 & 0.0246 \\
POMS & 797.1 & 3954 & 0.9346 & 0.0195 \\
\hline
\end{tabular}


Table III. Results of $P V T$ calculations by Flory's equation of state theory

\begin{tabular}{lcccl}
\hline Material & $T^{*} / \mathrm{K}$ & $P^{*} /$ bar & $V^{*} / \mathrm{cm}^{3} \mathrm{~g}^{-1}$ & $\begin{array}{l}\text { Volume } \\
\% \text { error }\end{array}$ \\
\hline PMMA & 8278 & 6007 & 0.7311 & 0.00524 \\
PcMA & 8047 & 5522 & 0.7827 & 0.00868 \\
PnBMA & 7046 & 5437 & 0.8150 & 0.00987 \\
BPE & 7188 & 4717 & 1.018 & 0.0127 \\
LPE & 7037 & 5199 & 1.005 & 0.0186 \\
PVA & 6454 & 6168 & 0.7093 & 0.00836 \\
PS & 8601 & 4461 & 0.8362 & 0.0171 \\
POMS & 8847 & 4738 & 0.8531 & 0.0146 \\
\hline & & & Average \% error $=0.0119$ \\
\hline
\end{tabular}

Table IV. Results of $P V T$ calculations by the cell theory

\begin{tabular}{lcccl}
\hline Material & $T^{*} / \mathrm{K}$ & $P^{*} /$ bar & $V^{*} / \mathrm{cm}^{3} \mathrm{~g}^{-1}$ & $\begin{array}{l}\text { Volume } \\
\% \text { error }\end{array}$ \\
\hline PMMA & 4717 & 7465 & 0.7920 & 0.00591 \\
PcMA & 4594 & 6740 & 0.8483 & 0.0111 \\
PnBMA & 4012 & 7171 & 0.8824 & 0.00861 \\
BPE & 4149 & 5260 & 1.108 & 0.00911 \\
LPE & 4091 & 5510 & 1.097 & 0.0160 \\
PVA & 3676 & 7710 & 0.7682 & 0.00541 \\
PS & 4910 & 5907 & 0.9059 & 0.0101 \\
POMS & 5051 & 5977 & 0.9245 & 0.0105 \\
\hline
\end{tabular}

Average $\%$ error $=0.00959$
Table V. Results of $P V T$ calculations by the Hole theory

\begin{tabular}{lcccl} 
Material & $T^{*} / \mathrm{K}$ & $P^{*} /$ bar & $V^{*} / \mathrm{cm}^{3} \mathrm{~g}^{-1}$ & $\begin{array}{l}\text { Volume } \\
\% \text { error }\end{array}$ \\
\hline PMMA & 11796 & 9284 & 0.8346 & 0.00588 \\
PcMA & 11431 & 8459 & 0.8929 & 0.0105 \\
PnBMA & 10143 & 8671 & 0.9323 & 0.00855 \\
BPE & 9961 & 6975 & 1.155 & 0.00803 \\
LPE & 9567 & 7543 & 1.133 & 0.0133 \\
PVA & 9208 & 9561 & 0.8100 & 0.00550 \\
PS & 12405 & 7145 & 0.9569 & 0.0110 \\
POMS & 12661 & 7388 & 0.9749 & 0.0105 \\
\hline
\end{tabular}

Average $\%$ error $=0.00917$

Table VI. Parameters of the Tait equation for calculating experimental isothermal compressibility

\begin{tabular}{lccc}
\hline Material & $B_{0} /$ bar & $B_{1} \times 1000 /{ }^{\circ} \mathrm{C}^{-1}$ & Volume \% error \\
\hline PMMA & 2929 & 4.329 & 0.00306 \\
PcMA & 2795 & 4.986 & 0.00435 \\
PnBMA & 2203 & 4.953 & 0.00217 \\
BPE & 1735 & 4.621 & 0.00475 \\
LPE & 1763 & 4.674 & 0.00559 \\
PVA & 2025 & 4.153 & 0.00607 \\
PS & 1841 & 2.201 & 0.00801 \\
POMS & 2573 & 4.133 & 0.0115 \\
\hline
\end{tabular}

Average $\%$ error $=0.00569$

Table VII. \% error in thermal expansivity and isothermal compressibility of each theory

\begin{tabular}{|c|c|c|c|c|c|c|c|c|c|c|}
\hline & \multicolumn{2}{|c|}{ This work } & \multicolumn{2}{|c|}{$\begin{array}{l}\text { Flory's equation } \\
\text { of state theory }\end{array}$} & \multicolumn{2}{|c|}{$\begin{array}{l}\text { Lattice fluid } \\
\text { theory }\end{array}$} & \multicolumn{2}{|c|}{ Cell theory } & \multicolumn{2}{|c|}{ Hole theory } \\
\hline & $\begin{array}{l}\% \text { error } \\
\text { in } \alpha\end{array}$ & $\begin{array}{l}\% \text { error } \\
\text { in } \beta\end{array}$ & $\begin{array}{l}\% \text { error } \\
\quad \text { in } \alpha\end{array}$ & $\begin{array}{l}\% \text { error } \\
\text { in } \beta\end{array}$ & $\begin{array}{l}\% \text { error } \\
\text { in } \alpha\end{array}$ & $\begin{array}{l}\% \text { error } \\
\text { in } \beta\end{array}$ & $\begin{array}{l}\% \text { error } \\
\text { in } \alpha\end{array}$ & $\begin{array}{l}\% \text { error } \\
\text { in } \beta\end{array}$ & $\begin{array}{l}\% \text { error } \\
\text { in } \alpha\end{array}$ & $\begin{array}{c}\% \text { error } \\
\text { in } \beta\end{array}$ \\
\hline PMMA & 1.35 & 0.949 & 1.88 & 2.40 & 3.77 & 4.13 & 2.16 & 1.17 & 1.29 & 0.803 \\
\hline PcMA & 1.75 & 1.62 & 1.94 & 2.37 & 3.71 & 3.88 & 2.56 & 1.80 & 1.64 & 1.54 \\
\hline BPE & 1.35 & 0.0858 & 2.14 & 2.68 & 3.08 & 3.62 & 2.20 & 0.705 & 0.972 & 0.844 \\
\hline LPE & 1.78 & 0.365 & 2.57 & 2.46 & 3.05 & 2.94 & 2.89 & 0.895 & 1.03 & 1.15 \\
\hline PVA & 1.26 & 0.353 & 1.76 & 2.51 & 2.96 & 3.86 & 1.57 & 0.503 & 1.25 & 0.375 \\
\hline PS & 1.28 & 1.12 & 2.73 & 3.97 & 4.84 & 6.16 & 1.56 & 1.00 & 1.54 & 1.26 \\
\hline POMS & 1.26 & 1.05 & 1.51 & 2.92 & 3.37 & 4.62 & 1.86 & 1.28 & 1.19 & 0.891 \\
\hline Average $\%$ error & 1.44 & 0.859 & 2.12 & 2.79 & 3.79 & 4.39 & 2.17 & 1.14 & 1.33 & 0.962 \\
\hline
\end{tabular}

correlation, the Tait equation, ${ }^{8,9}$ as follows.

$$
\frac{V(t, P)}{V\left(t, P^{0}\right)}=1-C \ln \left[1+\frac{P-P^{0}}{B_{0} \exp \left(-B_{1} t\right)}\right]
$$

where $B_{0}$ and $B_{1}$ are experimental constants, $C$ is 0.0894 , $P$ is pressure in bars and $P^{0}$ is a reference pressure, 1 bar in this work. The parameters of the Tait equation for each polymer, determined in the same manner as eq 10 , are listed in Table VI. Isothermal compressibility $\beta$ from the Tait equation is,

$$
\beta=\frac{V(t, P)}{V\left(t, P^{0}\right)} \frac{C}{P-P^{0}+B_{0} \exp \left(-B_{1} t\right)}
$$

Using the parameters in Tables $\mathrm{I}$ to $\mathrm{V}$, theoretical values for thermal expansivity and isothermal compressibility were calculated and compared with experimental values from eq 11 and 13. The results are listed in Table VII. Equation 7 gives similar precision to the Hole theory and better results than other theories as in the $P V T$ data. But, the theoretical values for $\alpha$ and $\beta$, as a whole, are not in good agreement with the experimental values, unlike $P V T$ data. One main reason for poor agreement with the experimental values in thermal expansivity and isothermal compressibility may be that the potential field of eq 5 is not proper for polymeric liquids although it is seen to be quantitatively better than the van der Waals potential of the LF theory.

\section{CONCLUSION}

In this work, the lattice for describing the polymeric state was so finely divided that the sites on the lattice became continuously distributed. Thus, unlike the old 
lattice theory, volume dependent potential can be used. This continuous lattice model with van der Waals potential produced the same equation of state as the original LF Theory. The calculated $P V T$ data by the modified model with the Lennard-Jones 6-12 potential were in better agreement with experimental $P V T$ data than other theories except the Hole theory, but in poor agreement with experimental values for $\alpha$ and $\beta$ as in other theories. For improvement of $\alpha$ and $\beta$, a better potential field should be introduced.

\section{REFERENCES}

1. P. J. Flory, "Principles of Polymer Chemistry," Cornell Uni- versity Press, Ithaca, New York, 1953, Chapter 12.

2. I. C. Sanchez and R. H. Lacombe, J. Phys. Chem., 80, 2352 (1976)

3. I. C. Sanchez and R. H. Lacombe, J. Polym. Sci., Polym. Lett. Ed., 15, 71 (1977)

4. G. T. Dee and D. J. Walsh, Macromolecules, 21, 811 (1988).

5. D. J. Walsh, G. T. Dee, J. L. Halary, J. M. Ubiche, M. Millequant, J. Lesec, and L. Monnerie, Macromolecules, 22, 3395 (1989).

6. R. Simha and T. Somcynsky, Macromolecules, 2, 342 (1969).

7. P. J. Flory, R. A. Orwoll, and A. J. Vrij, J. Am. Chem. Soc., 86, 3507 (1964).

8. A. Quach and R. Simha, J. Appl. Phys., 42, 4592 (1971).

9. O. Olabisi and R. Simha, Macromolecules, 8, 206 (1975).

10. J. E. McKinney and M. Goldstein, J. Res. Natl. Bur. Stand. A, 78, 331 (1974). 